\title{
An Attributable Role-Based Access Control for Healthcare
}

\author{
Dirk Schwartmann \\ German Aerospace Center (DLR), Cologne, Germany \\ Dirk.Schwartmann@dlr.de
}

\begin{abstract}
Role Based Access Control (RBAC) has the potential for reducing the complexity and total cost of security administration. Even though RBAC implementations aim on administrating large scale systems, they have a shortcoming in common. They do not allow to define attributable roles and permissions. But such roles are very common in our thoughts and language. When we say "attending physician of patient $\mathrm{x}$ ", we mean a role attending physician with all associated permissions to fulfill the treatment of patient $\mathrm{x}$. Because the resulting permissions only differ in the restriction to a particular patient, it is desirable that attributes like "patient x" are used in roles and permissions to restrict the rights to access only data related to that patient. This paper shows how attributes can be applied to RBAC, in order to reduce the total number of role- and permission-objects in security administration.
\end{abstract}

\section{Introduction}

One of the most challenging problems in modern healthcare is the security administration of large networked systems containing patient data. Today's security administration is usually based upon access control lists for each user, leading to large time exposures for updating access control lists on the data, creating new and deleting old users.

Role Based Access Control (RBAC) has the potential to reduce the complexity and total cost of security administration. Access Rights are defined by User-toRole and Role-to-Permission assignments. This reduces the need to administrate rights at data level (e.g. files on a file-system). Even though RBAC implementations aim on administrating large scale systems, they have a shortcoming in common: they do not allow to define attributable roles and permissions. However, such roles are very common in our thoughts and language. We say "attending physician of patient a" and "quality assurance officer in project x". It is desirable that attributes like "patient a" restrict the rights to access only data related to that patient to fulfill the least-rights-principle. That means the user should possess the minimum rights to fulfill the current task. A static role "attending physician" alone would break this rule. A person in this role would have access to data of all patients. So, the problem must be solved in a different way. The simple solution would be the introduction of a static role "attending physician of patient $\mathrm{x}$ " with appropriate permissions to restrict access to the data of this 
patient within all patient records. But this is not a very good idea. Assume the amount of administration in a large hospital with hundreds of patients. For each new patient a new attending physician role would be created.

In August 2001 the National Institute of Standards and Technology (NIST) published a proposed standard for RBAC that includes the fundamental set of RBAC components (see [1]). In this paper an extension to the standard with attributable permissions and roles will be introduced. At the time of role activation the attribute values are passed on to it and are subject to permission checks. So, different attribute values lead to different access rights. As a result a single attributable role "attending physician" will be enough for a hospital. Thus, the amount of roles and permissions is reduced dramatically resulting in less error-prone administration and in an enormous reduction of the total costs of security administration.

\section{Overview of RBAC}

Role-based access control (RBAC) is a proven alternative to traditional access control mechanisms like discretionary access control (DAC) and mandatory access control (MAC). RBAC allows to represent the natural organizational view upon access rights. Roles are used to describe the functions of individuals with associated permissions. In RBAC, permissions are defined as operations on objects. Users are assigned to roles and are granted permissions this way. During sessions users activate roles to fulfill job functions with distinct rights.

Figure 1 gives an overview of the conceptual items and their interrelations in hierarchical RBAC of the proposed NIST standard. The hierarchical RBAC extends the so-called core RBAC with role hierarchies. General role hierarchies provide support for an arbitrary partial order to serve as the role hierarchy which models inheritance of permissions and, in the opposite direction, user membership among roles. Because it is needed further down in this paper the definition of general role hierarchies of the proposed NIST standard is presented here:

\section{Definition 1. General Role Hierarchies}

$R H \subseteq R O L E S \times R O L E S$ is a partial order on ROLES called inheritance relation, written as $\succeq$, where $r_{1} \succeq r_{2}$ only if all permissions of $r_{2}$ are also permissions of $r_{1}$, and all users of $r_{1}$ are also users of $r_{2}$.

Formally: $r_{1} \succeq r_{2} \Rightarrow$ authorized_permissions $\left(r_{2}\right) \subseteq$ authorized_permissions $\left(r_{1}\right) \wedge$ authorized_users $\left(r_{1}\right) \subseteq$ authorized_users $\left(r_{2}\right)$

\section{Authorization on Life-Long Health-Records}

The aim of health-records for every single patient that are to be used in shared care throughout the whole life of each person, bears some problems. Envisioning the existence of a (distributed) electronic health record for each living person, the authorization mechanism must allow access to each patient-record in a treatment case. It is clear that this cannot be fulfilled by introducing a static role "treating physician of patient $x$ " for each patient $\mathrm{x}$ in practice. The number of roles in 


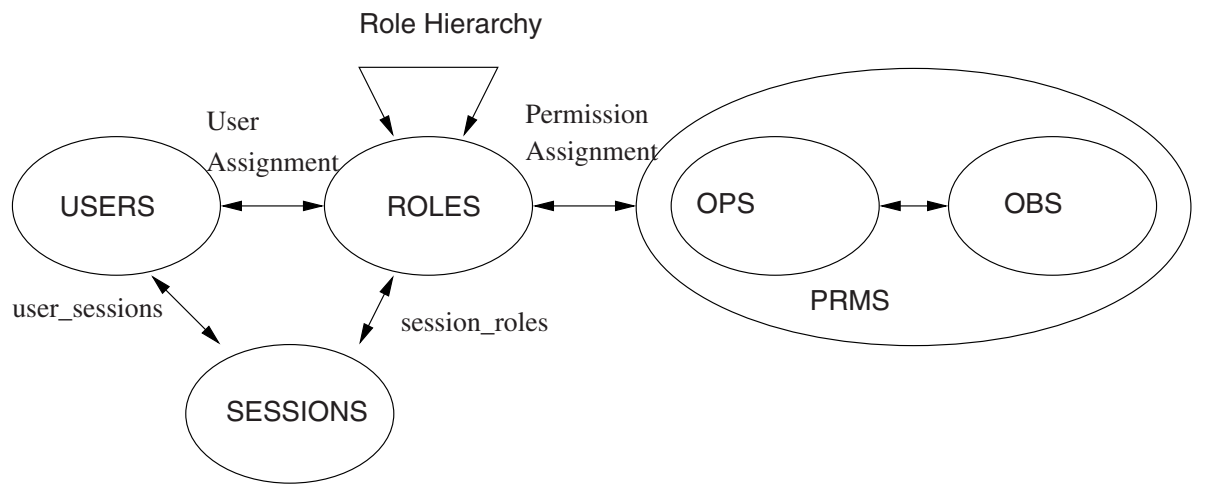

Fig. 1. Hierarchical RBAC

IT-systems would steadily increase. As a consequence security administration would become a difficult task and no matter how efficient concrete authorization sub-systems would be implemented, the large number of roles would have a very negative impact on the time required to make concrete permission decisions. Introducing a single role "treating physician" to permit access to every healthrecord is not a solution. Not only the possible infringement of a person's individual rights through access to a (distributed) life-long health record outside a treatment case is problematic. From the sight of security this role is in fact a great danger. An unauthorized person that possesses such a role could access healthcare data of every living individual.

\section{Attributable Permissions and Roles in RBAC}

In this section an attributable version of RBAC will be introduced. Attributedefinitions associated to permission objects are used to define classes of permissions. The assignment of permissions with attribute-definitions to roles constrains role activation. During activation within a session conforming Attribute-Values must be given in a role-activation-context. The attribute-values are evaluated by the permissions during run-time. Figure 2 gives an overview of the connections between the conceptual items in the RBAC-Extension introduced herein. In the following sections the items of the concept are discussed.

\subsection{Attribute-Definitions and Attribute-Values}

An attribute-definition holds the unique name and type of the attribute within the authorization subsystem. An attribute-definition can be shared among several permissions. Typical examples for attribute-definitions in healthcare would be patient-identification and treatment-case. Permissions with attributedefinitions allow to define classes of permissions analogous to classes in objectoriented design. This approach reduces the number of permission objects to administrate and allows to introduce conforming attribute values. 


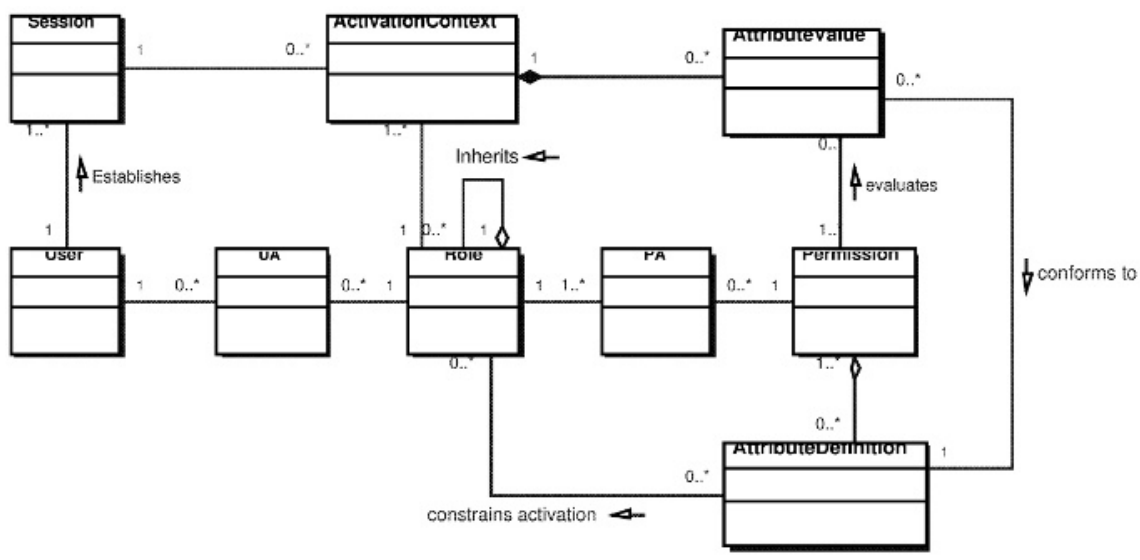

Fig. 2. Extended RBAC with Attributes

\section{Definition 2. Attribute-Definition}

An attribute-definition must allow at least a single attribute-value. As a consequence the set of possible attribute-values for an attribute-definition must not be empty.

An attribute-value simply consists of an association to an attribute-definition and of a value conforming to that definition. Formally, the set of all attributedefinitions and all attribute-definitions of a permission is defined as follows:

Definition 3. Set of all attribute-definitions

Let $A D$ be all attribute-definitions of the authorization sub-system.

Definition 4. Attribute-definitions of a permission $A D P: P R M S \rightarrow 2^{A D}$, $\forall p \in P R M S: A D P(p)=\{a d \in A D \mid$ ad is part of aggregate $p\}$

\subsection{Activation-Context}

Activation of a role is constrained by all attribute-definitions that is implicitly attached to it through permission assignments.

Definition 5. Direct attribute-definitions of a role $D A D R: R O L E S \rightarrow 2^{A D}$, $\forall r \in R O L E S: D A D R(r)=\{a d \in A D \mid a d \in A D P(p) \wedge(p, r) \in P A\}$

Also all attribute-definitions of inherited roles are inherited and thus become attributes of a role. Now the complete set of attribute-definitions of a role can be defined:

Definition 6. Attribute-Definitions of a Role $A D R: R O L E S \rightarrow 2^{A D}$, $\forall r \in R O L E S: A D R(r)=\bigcup_{r^{\prime} \in R O L E S: r \succeq r^{\prime}} D A D R\left(r^{\prime}\right)$ 
Upon construction this set holds all attribute-definitions from the role hierarchy following the role inheritance structure as defined by the general role hierarchy of the proposed RBAC standard.

Role activation then looks like this: for each attribute-definition of a role exactly one conforming attribute value must be given in the activation-context. Sets are possible through multiple instances of activation-contexts. For example, to allow a physician to be attending physician of patients $\mathrm{A}, \mathrm{B}$ and $\mathrm{C}$, an activation-context is created for each patient. Activation-contexts serve only a single role and are created and filled before that role can be activated. Activation of a role requires that the activation-context holds attribute-values for all attribute-definitions of that role. Formally the set of attribute-values in an activation context and the constraints on role activation are defined as follows:

Definition 7. Attribute-values of an attribute-definition $\forall a d \in A D$ let $A V(a d)$ be the (non-empty) set of all possible attribute-values of ad.

Definition 8. Activation contexts of a role in a session $\forall s \in S E S S S I O N S \forall r \in R O L E S: A C_{s}(r):=$ Set of activation-contexts for role $r$ in session $s$.

Definition 9. Attribute-values of activation-contexts for a role $r$ in session $s$ $\forall a c \in A C_{s}(r) \forall a d \in A D$ :

value $_{s, r}(a c, a d)= \begin{cases}a v \in A V(a d) & \text { if } a d \in A D R(r) \text { and ac has value for ad } \\ \emptyset & \text { if ad } \in A D R(r), \text { but ac has no value for ad } \\ \emptyset & \text { otherwise }\end{cases}$

Definition 10. Activation constraint on roles

Let ac be an activation-context for role $r$ The role $r$ can be activated in a session $s$ if and only if:

$\exists a c \in A C_{s}(r) \forall a d \in A D R(r):$ value $_{s, r}(a c, a d) \neq \emptyset$

A role can only be activated within activation-contexts that fulfill the last definition.

\subsection{Restriction of Attribute-Values}

In most cases the security policy requires that the possible attribute-values shall be restricted. The user-assignment object can hold a list of allowed attributevalues for each attribute-definition of a role. The list may also be inverted to define only forbidden ones, or a combination of both lists can be used. For example, the role "emergency-doctor" would not restrict access to health-records of patients, while the user-assignment object of the role "attending physician" might hold only the IDs of the patients which the physician is currently treating. 
The restriction on attribute-values using the user-assignment object is easy and still very powerful. Sophisticated implementations might use X509 attribute certificates [4] to define the allowed value-set. An approach to use such certificates for RBAC is already existing in another project that uses attribute certificates to administrate RBAC completely [3].

\subsection{Role-Delegation in Attributable RBAC}

The delegation of roles from one user to another requires two steps. First, a userassignment must be created. Second, the according attribute-value-lists must be copied from the delegating user's user-assignment object to the user-assignment object of the delegee.

\subsection{Checking Permissions in Attributable RBAC}

To check permissions within a specific session the authorization engine uses the authorization-context(s) of activated roles. The algorithm is as easy as follows:

1. Retrieve all activation-contexts from the session that belong to activated roles. The set shall be Activated $A C_{s}$. continue with

2. For each activation-context $a c \in$ Activated $A C_{s}$ do

a) Get role from $a c$. Let $r$ be the role.

b) Get assigned permissions for role $r$. Let $P A$ the set of assigned permissions.

c) For each $p \in P A$ do

i. Get all attribute-definitions from $p$. Let $D$ be this set.

ii. For all $d \in D$ retrieve the value from $a c$ building a set $V$.

iii. use set $V$ to evaluate the permission upon the information space

The difference between standard RBAC permission checks and the algorithm above is that one has to retrieve the values from the activation context before the permission can be evaluated upon the information space. But the values can easily be cached at the permission objects after the first time. Compared to regular permission objects only one indirection is still necessary, then. This is because several activation-contexts can share a permission object with different values assigned. Because the computational cost of this is constant (pointer arithmetic) this indirection can be neglected in practice.

\section{Example Reducing Role- and Permission-Objects}

Let us consider a small part of a role hierarchy in an hospital that is conforming to the least-privilege-principle and uses static roles and permissions. That means roles and permission without attribute-definitions, as introduced herein. In specific the least-privilege-principle implies that there would be roles and permissions for each single patient. Figure 3 shows this situation.

With the attributable RBAC approach introduced in this paper the roles and permissions reduce to only two instances as shown in Fig. 4. The benefit for 


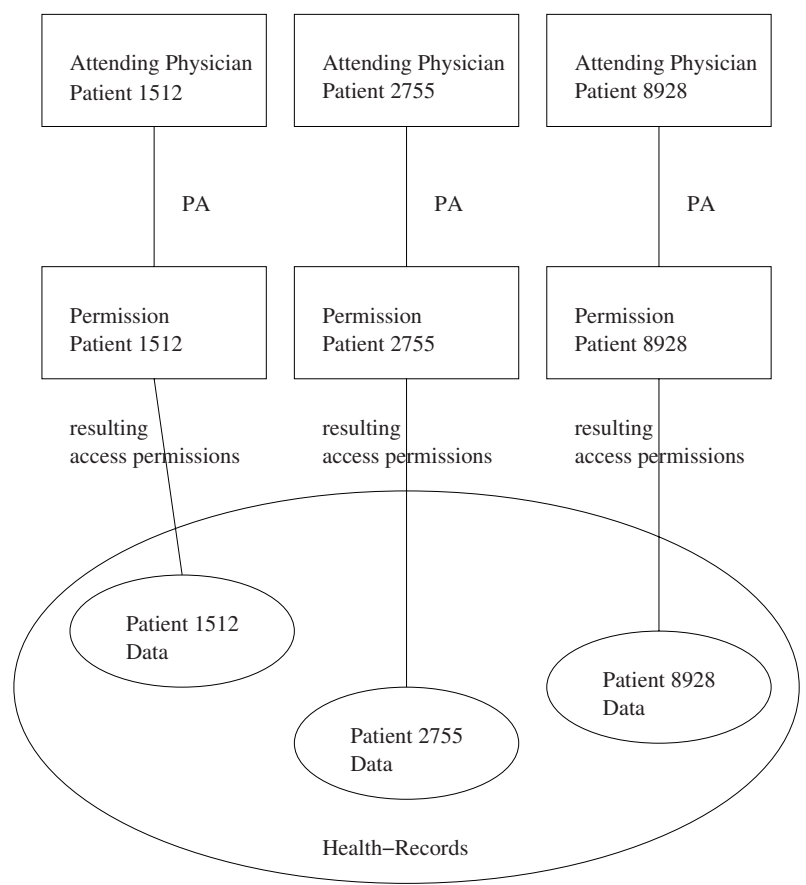

Fig. 3. Extended RBAC with Attributes

security administration becomes clear if one thinks about a large hospital with thousands of health-records for patients. If for example a revision of the hospital's security-policy would require to change the permission objects of all patients then either an administrative batch job would be necessary to adapt the permission objects of all patients or it would take a very long time to update the authorization rules. With attributable RBAC such changes would only affect a small number of objects. In the example case only one object compared to thousands.

\section{Summary and Outlook}

The extension of RBAC with attributes on permissions and roles is very effective. In large-scale systems the usually extremely high number of permissions and roles can be reduced to a much smaller amount which is easier to administrate and thus less error-prone.

Another advantage of the attributable RBAC is that it is ready for working together with attribute certificates. The newest health professional cards in healthcare are already smartcards with X.509 certificates and asymmetric key pair. This will allow physicians to generate valid attribute certificates holding attribute-values according to attributable RBAC as described here. Many tasks can easily be done then. The implementation of referals, second opinions, de- 


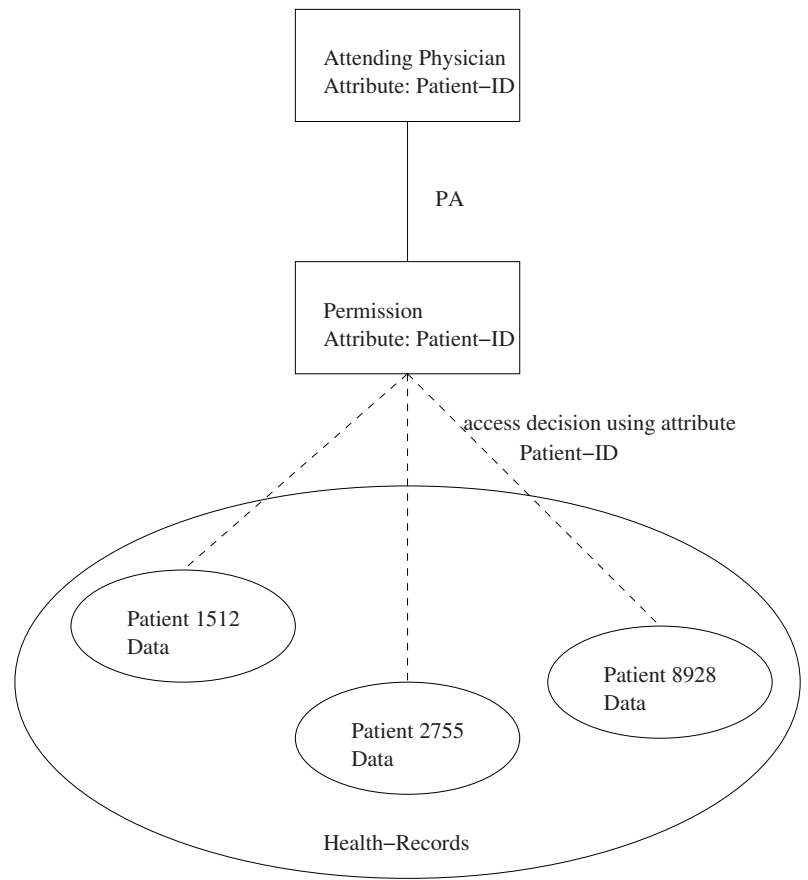

Fig. 4. Reducing role- and permission-objects with attributable RBAC

legation of cases, etc. can all be done with the same technical approach using secure attribute-certificates without the need to administrate the authorization rules by hand.

\section{References}

1. David F. Ferraiolo and Ravi Sandhu and Serban Gavrila and D. Richard Kuhn and Ramaswamy Chandramouli: Proposed NIST Standard for Role-Based Access Control. ACM Transactions on Information and System Security, Vol. 4 (2001) $224-274$

2. W. Essmayr and E. Kapsammer and R.R. Wagner and A.M. Tjoa: Using RoleTemplates for Handling Recurring Role Structures. 12th IFIP WG 11.3 Conference on Database Security, Chalkidiki, Greece, July 15-17, 1998

3. D.W.Chadwick, O.Otenko: The PERMIS X.509 Role Based Privilege Management Infrastructure ISI, University of Salford, Salford, M5 4WT

4. Farrell, S., and R. Housley: An Internet Attribute Certificate Profile for Authorization, RFC 3281, IETF, April 2002. 